\title{
Coffee Consumption and C-Reactive Protein Levels: A Systematic Review and Meta-Analysis
}

\author{
Elizabeth D. Moua ${ }^{1}$, Chenxiao Hu ${ }^{2}$, Nicole Day ${ }^{3}$, Norman G. Hord ${ }^{4}$ and Yumie Takata ${ }^{4, *}$ \\ 1 College of Pharmacy, Oregon State University/Oregon Health \& Science University, Corvallis, OR 97331, \\ USA; mouae@oregonstate.edu \\ 2 Department of Statistics, College of Science, Oregon State University, Corvallis, OR 97331, USA; \\ huche@oregonstate.edu \\ 3 College of Engineering, School of Chemical, Biological, and Environmental Engineering, Oregon State \\ University, Corvallis, OR 97331, USA; Nicole.B.Day@colorado.edu \\ 4 College of Public Health and Human Sciences, School of Biological and Population Health Sciences, \\ Oregon State University, Corvallis, OR 97331, USA; norman.hord@oregonstate.edu \\ * Correspondence: yumie.takata@oregonstate.edu; Tel.: +1-541-737-1606
}

Received: 23 March 2020; Accepted: 6 May 2020; Published: 8 May 2020

\begin{abstract}
Coffee contains bioactive compounds with anti-inflammatory properties, and its consumption may reduce c-reactive protein (CRP) levels, a biomarker of chronic inflammation. A previous meta-analysis reported no overall association between blood CRP level and coffee consumption by modeling the coffee consumption in categories, with substantial heterogeneity. However, the coffee cup volume was not considered. We conducted a systematic review and dose-response meta-analysis investigating the association between coffee consumption and CRP levels reported in previous observational studies. A dose-response meta-analysis was conducted by mixed-effects meta-regression models using the volume of coffee consumed as metric. Eleven studies from three continents were identified using the PubMed database, totaling 61,047 participants. Three studies with the largest sample sizes observed a statistically significant association between coffee and CRP levels, which was inverse among European and United States (US) women and Japanese men (1.3-5.5\% decrease in CRP per $100 \mathrm{~mL}$ of coffee consumed) and positive among European men (2.2\% increase). Other studies showed no statistically significant associations. When all studies were combined in the dose-response meta-analysis, no statistically significant associations were observed among all participants or when stratified by gender or geographic location, reflecting the conflicting associations reported in the included studies. Further studies are warranted to explore these inconsistent associations.
\end{abstract}

Keywords: coffee consumption; c-reactive protein; cross-sectional studies; systematic review and meta-analysis

\section{Introduction}

Coffee is a well-known beverage around the world and the most popular caffeinated drink choice [1,2]. A recent meta-analysis of 31 cohort studies reported that coffee consumption is associated with decreased risk of total mortality and cause-specific mortality from cardiovascular disease (CVD) and cancer [3]. C-reactive protein (CRP) is considered a biomarker of chronic inflammation [4] and of disease risk and progression [5], including for CVD. The association between coffee and mortality reported in the meta-analysis may be mediated through CRP.

Coffee contains many bioactive components such as chlorogenic acids, polyphenols, diterpenes, micronutrients and caffeine [2,6-8], which may exert beneficial health effects through antioxidant and 
anti-inflammatory properties [6-8]. There are also bioactive compounds that may negatively affect health; for instance, the high amount of lipids in unfiltered coffee may increase blood cholesterol $[9,10]$.

In this analysis, we investigated the associations between coffee consumption and CRP levels among adults in observational studies by conducting a systematic literature review and a dose-response meta-analysis. A previous meta-analysis of 24,863 participants from nine studies modeled coffee consumption by categories and reported no overall statistically significant association with CRP level, but there was evidence of substantial heterogeneity [11]. The cup volume varied by geographic location, which affects the amount of bioactive compounds consumed and, consequently, their biological effects $[7,8,12-14]$. Hence, we modeled the volume of coffee consumed and hypothesized that higher coffee consumption is associated with lower levels of CRP.

\section{Materials and Methods}

\subsection{Registration}

This study was reported according to the Preferred Reporting Items for Systematic Reviews and Meta-Analyses (PRISMA) [15] and is registered with the PROSPERO International Prospective Register of Systematic Reviews (CRD42018108351).

\subsection{Literature Search}

A systematic literature review was conducted using the electronic database PubMed to collect data from published studies. The following search terms were used: ("coffee" [MeSH Terms] OR "coffee"[All Fields]) AND ("c-reactive protein"[MeSH Terms] OR ("c-reactive"[All Fields] AND "protein"[All Fields]) OR "c-reactive protein"[All Fields] OR “c reactive protein"[All Fields]). The last search was conducted in March 2020, and a total of 61 abstracts were identified and reviewed independently by two authors (E.D.M. and Y.T.). A full-text article was not obtained if the title and/or abstract met one or more of the following exclusion criteria: (1) animal study; (2) study among children; (3) no mention of coffee or other beverages, or of associations between food or beverage intake other than coffee and CRP or other inflammatory biomarkers; (4) no mention of CRP or other inflammatory markers/biomarkers, or of associations between beverage consumption and outcome variables other than CRP; (5) non-original study such as review or commentary. After removing abstracts that met those exclusions, the full-text articles of the remaining abstracts were obtained, reviewed, and excluded if they met one of the following additional exclusion criteria: (1) not reporting the association between coffee consumption and CRP level; (2) overlapping study populations; (3) not reporting enough data, such as coffee consumption categories (at least three) and levels of CRP within those categories; (4) meeting any of the previous exclusion criteria that could not be determined from the abstract alone, but was determined based on the full-text article. A total of 10 articles that did not meet any of the exclusion criteria were included in the systematic literature review. We found three meta-analysis/systematic review articles in our PubMed search $[4,11,16]$ and compared the list of original research studies included in the article, finding no additional studies to the 10 articles we identified that met our inclusion criteria. Data were extracted independently by two authors (E.D.M. and Y.T.) and inconsistencies were discussed and brought to consensus.

The following information was extracted, compiled and summarized for each study: first author; year of publication; study name; country; study design; calendar years when the study was conducted and questionnaire information and blood samples were collected; number of participants; age; gender; coffee consumption assessment (questionnaire validated or not) and methods of questionnaire administration (interview or self-administered); cup volume conversion and/or frequency of consumption; study results such as CRP levels (mean and standard deviations/error or $95 \%$ confidence intervals (CI)) by coffee consumption categories.

Not all of the information described above could be extracted from each of the 10 articles. In such cases, articles that might provide missing information were examined by examining references cited 
by the studies, searching for articles about the study or questionnaire through PubMed, or contacting the corresponding author of the articles. For instance, information on cup volume conversion was available for nine studies [17-24], but not for the Kyushu University cohort and the Dose-Response to Exercise in Women (DREW) trial $[25,26]$. When we contacted the corresponding authors of these two studies, we either received confirmation of the lack of information [26] or no response [25]; hence, we estimated the cup conversion based on the other studies conducted in similar location and calendar years, such as the Aichi Workers' study [20] and Nurses' Health Study (NHS) [18], respectively. Data on high-sensitivity CRP levels were obtained from all studies, but two studies needed to be converted to milligram per liter [20,22]. For all 11 studies, high-sensitivity CRP was measured using blood samples collected throughout each study.

The risk of bias for each study was assessed by both authors (E.D.M. and Y.T.) independently using the modified Newcastle-Ottawa Scale for cross-sectional studies [27]; any discrepancies were discussed and a consensus reached.

\subsection{Meta-Analysis}

A meta-analysis of associations between coffee consumption volume and CRP level was conducted through the Metafor package from R. Based on the cup conversion information we collected and the range of cups of coffee consumed in each category reported in each article, we estimated the mid-point volume (in $\mathrm{mL}$ ) of coffee consumption in each category; we then re-calculated the $p$-trend to test a linear association between coffee consumption and CRP level by treating coffee volume as continuous in a model.

The estimated weighted mean changes in CRP level (per $100 \mathrm{~mL}$ of coffee) and 95\% CI were calculated through a mixed-effects meta-regression model. We used log-transformed CRP levels in our meta-analysis. Due to incomparability of the reported values, the ATTICA study was excluded from the meta-analysis. The heterogeneity across studies was assessed by $I^{2}$ statistics [28]. To explore the source of heterogeneity, we pre-specified and stratified analyses by gender and geographic location.

\section{Results}

Our study identified 61 abstracts published in March 2020 or earlier with search terms identified previously. After reviewing articles based on the title and abstract (Figure 1), 47 were selected to obtain full-text articles. Among them, 37 of the published studies were excluded: 26 for not reporting the association between coffee consumption and CRP level, four for using the same study population [29-32], five for not providing enough data [33-37], and two for being a non-original article [38,39]. In total, 10 published articles reporting associations between coffee consumption and CRP levels in 11 study populations (one article reported results from two cohorts [18]) were selected and included in the current systematic review and meta-analysis.

The 11 studies were conducted between 1976 and 2007 in various locations: three in the United States (US) [18,26], three in Asia [19,20,25], and the rest in Europe [17,21-24] (Table 1). These 11 studies involved a total of 61,047 participants. Most studies had over 500 participants each [17-20,22,23,25], with the exception of two studies which had 344 and 61 participants, respectively [21,26]. Among the 11 studies, six included both men and women [17,19,20,22,23,25]. Of the other five studies, two included only women $[18,26]$ and three included only men $[18,21,24]$. 


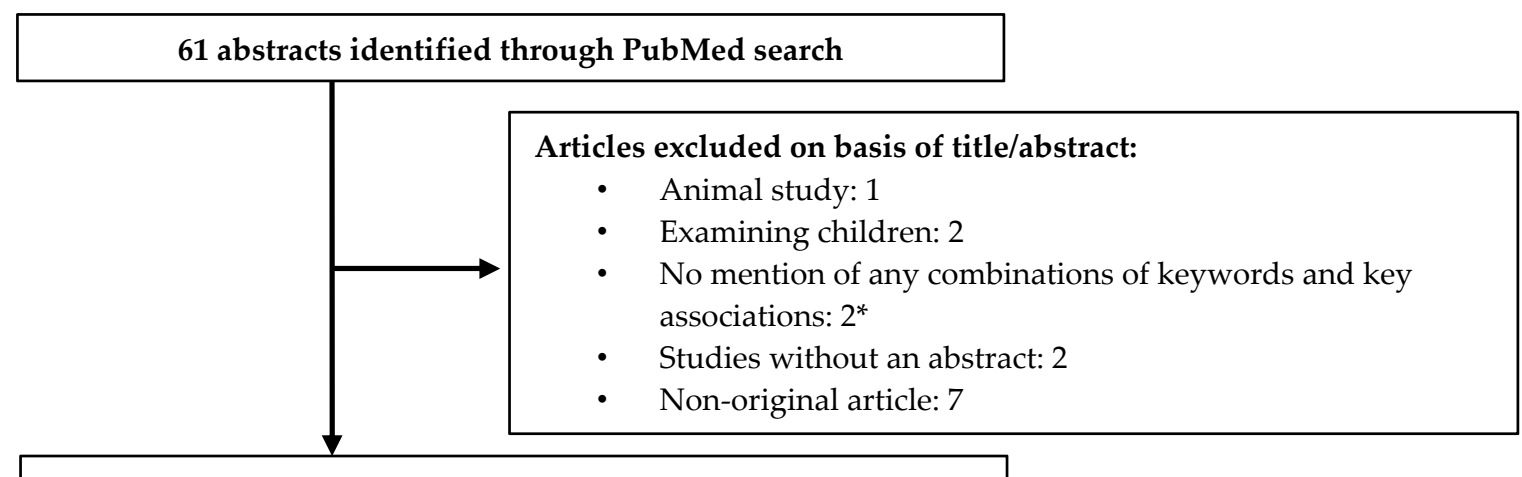

47 articles met inclusion criteria and full texts were obtained

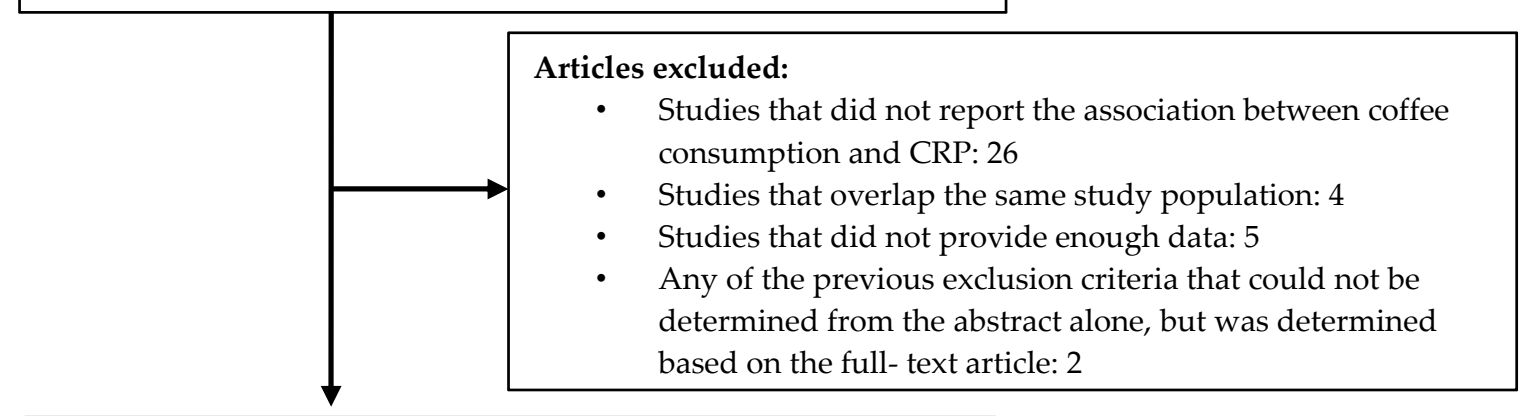

10 articles were included in systematic literature review

Figure 1. Flow chart of systematic literature review. * no mention of coffee or other beverages, or of associations between food or beverage intake other than coffee and c-reactive protein (CRP) or other inflammatory biomarkers; no mention of CRP or other inflammatory markers/biomarkers, or of associations between beverage consumption and outcome variables other than CRP.

The age of study participants varied from 18 to 87 years old. The mean body mass index (BMI) of each study was between $22.8 \mathrm{~kg} / \mathrm{m}^{2}$ and $36.1 \mathrm{~kg} / \mathrm{m}^{2}$, with the exception of one that did not report BMI values, but reported instead that $17.7 \%$ of participants were obese [17]. Six studies reported that the mean alcohol consumption ranged from 1.7-27.3 g/day [18,20-24,26], while four studies reported that $18.6-72.7 \%$ of participants drank alcohol [19,25]. One study did not provide information on alcohol consumption [17]. As for smoking, one study of women and one study of men consisted of only non-smokers $[21,26]$. Hence, the percentage of current smokers ranged from $0 \%$ (due to recruitment criterion) in the DREW trial and United Kingdom (UK) study [21,26] to 53.3\% in the ATTICA study [17]. Of the three studies that included postmenopausal women and reported hormone therapy use $[18,22,26]$, the proportion of users ranged from $25.9 \%$ in the European Prospective Investigation into Cancer and Nutrition (EPIC) study [22] to 46.5\% in the DREW trial [26].

All studies used questionnaires to assess coffee consumption, through self-administration, interview, or both. Each study had 3-5 coffee consumption categories including "no", "low", "medium", "high", and "very high" coffee consumption. The consumption amount considered "low" or "high" varied across studies, ranging from 1 cup/month to $<6$ cups/week for low consumption and 2 to $\geq 6$ cups/day for high consumption. Two studies included the fifth ("very high") consumption category as high as $\geq 7$ cups/day [22,25]. As the studies were conducted in various locations, the volume of a cup for each region ranged from $150 \mathrm{~mL}$ in the Aichi Workers' cohort in Japan [20] and the Finnish Diabetic Nephropathy (FinnDiane) study in Finland [23] to $237 \mathrm{~mL}$ in NHS and Health Professional Follow-Up Study (HPFS) studies in the US [18] and EPIC and UK studies in Europe [21,22]. 
Table 1. Participant characteristics by study *.

\begin{tabular}{|c|c|c|c|c|c|c|c|c|c|c|}
\hline Study Name & Study Years & Gender & $\begin{array}{l}\text { Number of } \\
\text { Participants }\end{array}$ & $\begin{array}{l}\text { Age (Years } \\
\text { Old) }\end{array}$ & BMI & $\begin{array}{l}\text { Current } \\
\text { Smokers } \\
(\%)\end{array}$ & Alcohol & $\begin{array}{c}\text { Hormone } \\
\text { Therapy } \\
\text { Use (\%) }\end{array}$ & Coffee Consumption & Cup Conversion \\
\hline DREW trial [26] & 2001-2005 & Women & 344 & $\begin{array}{l}57.1 \pm 6.4 \\
45-75\end{array}$ & $36.1 \pm 3.9 \mathrm{~kg} / \mathrm{m}^{2}$ & $0 \%$ & $\begin{array}{c}1.7 \pm 2.4 \\
\text { g/day }\end{array}$ & $46.5 \%$ & $\begin{array}{l}55.2 \% \text { consumed }<1 \\
\text { cup } / \text { day }\end{array}$ & $\begin{array}{l}\text { No data (used } \\
237 \mathrm{~mL} \text { ) }\end{array}$ \\
\hline NHS [18] & 1976 & Women & 15,551 & 57.3 & $25.5 \mathrm{~kg} / \mathrm{m}^{2}$ & $13.4 \%$ & $6 \mathrm{~g} /$ day & $41.3 \%$ & $\begin{array}{l}28.9 \% \text { consumed } \leq 1 \\
\text { cup/day }\end{array}$ & $1 \mathrm{cup}=237 \mathrm{~mL}$ \\
\hline HPFS [18] & 1986 & Men & 7397 & 62.4 & $25.7 \mathrm{~kg} / \mathrm{m}^{2}$ & $6.2 \%$ & $11.8 \mathrm{~g} /$ day & - & $\begin{array}{c}36.1 \% \text { consumed } \leq 1 \\
\text { cup/day }\end{array}$ & $1 \mathrm{cup}=237 \mathrm{~mL}$ \\
\hline \multirow[t]{2}{*}{ EPIC Study [22] } & \multirow[t]{2}{*}{ 1992-2000 } & Women & 10,520 & $\begin{array}{c}51.7 \\
(45.3-58.4)\end{array}$ & $24.2 \mathrm{~kg} / \mathrm{m}^{2}$ & $17 \%$ & 4.2 g/day & $25.9 \%$ & 300 mL/day & \multirow[t]{2}{*}{1 cup $=237 \mathrm{~mL}$} \\
\hline & & Men & 4280 & $\begin{array}{c}53.3 \\
(46.6-59.6)\end{array}$ & $26.3 \mathrm{~kg} / \mathrm{m}^{2}$ & $30 \%$ & $14.5 \mathrm{~g} / \mathrm{day}$ & - & $380 \mathrm{~mL} /$ day & \\
\hline \multirow{2}{*}{$\begin{array}{c}\text { Kyushu } \\
\text { University Cohort } \\
\text { Study [25] }\end{array}$} & \multirow{2}{*}{ 2004-2007 } & Women & 5918 & \multirow{2}{*}{$\begin{array}{c}62 \\
49-76\end{array}$} & $22.5 \mathrm{~kg} / \mathrm{m}^{2}$ & $6 \%$ & $27.1 \%$ & \multirow{2}{*}{ No data } & \multirow{2}{*}{ Median: 2 cups/day } & \multirow{2}{*}{$\begin{array}{c}\text { No data (used } 150 \\
\mathrm{~mL} \text { ) }\end{array}$} \\
\hline & & Men & 4407 & & $23.5 \mathrm{~kg} / \mathrm{m}^{2}$ & $32.4 \%$ & $72.7 \%$ & & & \\
\hline $\begin{array}{l}\text { ATTICA study } \\
\text { [17] }\end{array}$ & $2001-2002$ & $\begin{array}{l}\text { Women } \\
\text { Men }\end{array}$ & $\begin{array}{l}1528 \\
1514\end{array}$ & $\begin{array}{c}45.5 \pm 13 \\
18-87\end{array}$ & $\begin{array}{l}15.6 \% \\
19.7 \%\end{array}$ & $\begin{array}{l}45.5 \% \\
62.4 \%\end{array}$ & No data & No data & $\begin{array}{l}24 \% \text { consumed }<1 \text { cup/day } \\
9 \% \text { consumed }<1 \text { cup/day }\end{array}$ & $1 \mathrm{cup}=150 \mathrm{~mL}$ \\
\hline $\begin{array}{c}\text { Singapore } \\
\text { Prospective Study } \\
{[19]}\end{array}$ & 2003-2007 & Both & 4139 & $48.8 \pm 11.3$ & $23.2 \mathrm{~kg} / \mathrm{m}^{2}$ & $11.7 \%$ & $18.6 \%$ & No data & $40 \%$ consumed $<1$ cup $/$ day & 1 cup $=215 \mathrm{~mL}$ \\
\hline $\begin{array}{c}\text { Aichi Workers' } \\
\text { Cohort Study [20] }\end{array}$ & 2002 & Both & 3317 & $\begin{array}{c}47.6 \pm 7.1 \\
35-69\end{array}$ & $22.8 \pm 2.7 \mathrm{~kg} / \mathrm{m}^{2}$ & $29.6 \%$ & $14.2 \mathrm{~g} /$ day & No data & Median: 1 cup/day & $1 \mathrm{cup}=150 \mathrm{~mL}$ \\
\hline $\begin{array}{l}\text { United Kingdom } \\
\text { Study [21] }\end{array}$ & 2003-2004 & Men & 61 & 32.7 & $25.3 \mathrm{~kg} / \mathrm{m}^{2}$ & $0 \%$ & $14.7 \mathrm{~g} /$ day & No data & Mean: 1.1 cup/day & $1 \mathrm{cup}=237 \mathrm{~mL}$ \\
\hline $\begin{array}{c}\text { FinnDiane Study } \\
\text { [23] }\end{array}$ & 1997 & Both & 1040 & $46.7 \pm 0.4$ & $25.6 \mathrm{~kg} / \mathrm{m}^{2}$ & $13.3 \%$ & 2.7 g/day & No data & $\begin{array}{c}12.9 \% \text { consumed }<1 \\
\text { cup/day } \\
\text { Mean: } 3.8 \pm 2.8 \text { cups/day }\end{array}$ & 1 cup $=150 \mathrm{~mL}$ \\
\hline BELSTRESS [24] & 1994-1998 & Men & 1031 & 49.0 & $27.0 \mathrm{~kg} / \mathrm{m}^{2}$ & $34.9 \%$ & $27.3 \mathrm{~g} /$ day & - & $16 \%$ consumed $<1$ cup $/$ day & 1 cup $=150 \mathrm{~mL}$ \\
\hline
\end{tabular}

* Mean or mean \pm standard deviation; range; and/or median (25th-75th percentiles) are provided for age; mean, median, or percentage of obesity is provided for BMI; mean or percentage of alcoho con

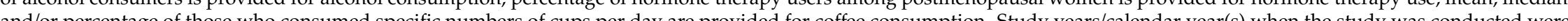
and/or pere obtaic collection procedures for cross-sectional study (ATTCA study [17]) or analysis of intervention trials or prospective studies (DREW [26], EPIC [22], Kyushu University cohort study [25], Singapore Prospective study [19], and Aichi Workers' study [20]); (2) extracting from another article of the same study (UK study [40] and the FinnDiane study [41,42] and BELSTRESS study [43]); or (3) extracting years when blood draw and coffee intake assessments through a food frequency questionnaire (FFQ) (NHS and HPFS [18]). Lifestyle factors and median (25th-75th percentiles) age by gender for EPIC were taken from other EPIC study publications [44,45], and lifestyle factors for BELSTRESS study were taken from another BESLTRESS study publication [46]. 
When the linear association was assessed taking the volume of coffee consumed into account, three of the 11 studies observed statistically significant inverse or positive associations between coffee consumption and CRP levels, although the rest reported no significant association (Table 2). These three studies examined associations separately by gender, and different associations were observed by gender and study [18,22,25]. Among women, the EPIC and NHS included 10,520 and 15,551 women, respectively, and they had a statistically significant inverse association (EPIC: $p$-trend $=0.002$, $2.7 \%$ decrease in CRP per $100 \mathrm{~mL}$ of coffee consumption; NHS: $p$-trend $=0.02,5.5 \%$ decrease in CRP) [18,22]. Among men, the Kyushu University cohort included 4407 men and also showed a statistically significant inverse association ( $p$-trend $=0.03,1.3 \%$ decrease in CRP) [25]. In contrast, the EPIC study had 4280 men and showed a statistically significant positive association between coffee consumption and CRP levels ( $p$-trend $=0.01,2.2 \%$ increase in CRP) [22].

When the 10 studies that reported compatible CRP levels were combined through the dose-response meta-analysis, no statistically significant association was observed among all participants (mean change in CRP level: $-2.4 \%$; 95\% CI: $-8.7 \%$ to $4.4 \% ; p=0.49$ ) with no evidence of heterogeneity $\left(I^{2}<0.01 \%\right)$. Similarly, no significant associations (mean change in CRP level; 95\% CI; $p$-value) were observed when we stratified by gender (men: $0.9 \% ;-9.7 \%$ to $8.8 \% ; p=0.85$; women: $-6.3 \% ;-16.4 \%$ to $5.0 \% ; p=0.26$ ), or geographic location (US: $-6.7 \% ;-6.5 \%$ to $8.3 \% ; p=0.54$; Europe: $0.6 \%$; $-6.5 \%$ to $8.3 \% ; p=0.83$; Asia: $-1.6 \% ;-12.1 \%$ to $10.2 \% ; p=0.78)$ with no evidence of heterogeneity $\left(I^{2}<0.01 \%\right)$. Given that BELSTRESS study [24] was based on crude estimates, we repeated the analyses without the BELSTRESS study, which did not materially change the results (data not shown).

Regarding risk of bias, assessed through the modified Newcastle-Ottawa Scale for cross-sectional studies [27], all studies had scores of six or higher (Supplementary Table S1). Five studies [20-23,25] scored seven, which is considered good quality. Two studies [24,26] scored six (satisfactory quality), three [18,19] scored eight (good quality), and another [17] scored 10 (very good quality). 
Table 2. C-reactive protein levels by coffee consumption by study.

\begin{tabular}{|c|c|c|c|c|c|c|c|}
\hline \multirow{3}{*}{ Study Name } & \multirow{3}{*}{ Gender } & \multicolumn{5}{|c|}{ Categories in the Original Study: Median or Mid-Point Volume $(\mathrm{mL})$} & \multirow{3}{*}{$P$-Trend * } \\
\hline & & None & Low & Medium & High & Very High & \\
\hline & & \multicolumn{5}{|c|}{$\begin{array}{c}\text { Number of Participants } \\
\text { Geometric Mean c-Reactive Protein (95\% Confidence Intervals) (mg/L) }\end{array}$} & \\
\hline \multirow{2}{*}{ DREW trial [26] } & & None: $0 \mathrm{~mL}$ & $\begin{array}{l}1 \text { cup/month to } 6 \\
\text { cups/week: } 104 \mathrm{~mL}\end{array}$ & $\begin{array}{c}1 \text { cup/day to } 13 \\
\text { cups/week: } 339 \mathrm{~mL}\end{array}$ & $\geq 2$ cups/day: $533 \mathrm{~mL}$ & - & \multirow[b]{2}{*}{0.05} \\
\hline & Women & $\begin{array}{c}104 \\
4.1(3.4,4.9)\end{array}$ & $\begin{array}{c}86 \\
4.1(3.3,5.0)\end{array}$ & $\begin{array}{c}89 \\
3.1(2.6,3.8)\end{array}$ & $\begin{array}{c}65 \\
3(2.4,3.8)\end{array}$ & - & \\
\hline \multirow[b]{2}{*}{ NHS [18] } & \multirow[b]{2}{*}{ Women } & None: $0 \mathrm{~mL}$ & $<1$ cup/day: $119 \mathrm{~mL}$ & 2-3 cups/day: $356 \mathrm{~mL}$ & $\geq 4$ cups/day: $1037 \mathrm{~mL}$ & - & \multirow[b]{2}{*}{0.02} \\
\hline & & $\begin{array}{c}3433 \\
4.18(3.55,4.92)\end{array}$ & $\begin{array}{c}4178 \\
4.04(3.60,4.52)\end{array}$ & $\begin{array}{c}5653 \\
3.25(3.03,3.48)\end{array}$ & $\begin{array}{c}2287 \\
2.37(2.16,2.59)\end{array}$ & - & \\
\hline HPFS [18] & Men & $\begin{array}{c}1723 \\
1.09(0.92,1.29)\end{array}$ & $\begin{array}{c}2341 \\
1.07(0.95,1.21)\end{array}$ & $\begin{array}{c}2354 \\
0.97(0.88,1.06)\end{array}$ & $\begin{array}{c}979 \\
1.00(0.88,1.12)\end{array}$ & - & 0.37 \\
\hline \multirow{3}{*}{ EPIC study [22] } & & None: $0 \mathrm{~mL}$ & Low: $103 \mathrm{~mL}$ & Medium-low: $297 \mathrm{~mL}$ & Medium-high: $451 \mathrm{~mL}$ & High: $745 \mathrm{~mL}$ & \multirow[b]{2}{*}{0.01} \\
\hline & Men & $\begin{array}{c}212 \\
1.15(1.13,1.16)\end{array}$ & $\begin{array}{c}1078 \\
1.16(1.15,1.18)\end{array}$ & $\begin{array}{c}977 \\
1.18(1.17,1.20)\end{array}$ & $\begin{array}{c}1078 \\
1.21(1.19,1.22)\end{array}$ & $\begin{array}{c}935 \\
1.34(1.33,1.36)\end{array}$ & \\
\hline & Women & $\begin{array}{c}832 \\
1.42(1.40,1.45)\end{array}$ & $\begin{array}{c}2730 \\
1.39(1.36,1.41)\end{array}$ & $\begin{array}{c}2171 \\
1.28(1.26,1.30)\end{array}$ & $\begin{array}{c}2730 \\
1.26(1.24,1.30)\end{array}$ & $\begin{array}{c}2057 \\
1.16(1.14,1.17)\end{array}$ & 0.002 \\
\hline \multirow{3}{*}{$\begin{array}{l}\text { Kyushu University } \\
\text { Cohort Study [25] }\end{array}$} & & None: $0 \mathrm{~mL}$ & $<1$ cup/day: $75 \mathrm{~mL}$ & 1-3 cups/day: $300 \mathrm{~mL}$ & 4-6 cups/day: 750 mL & $\geq 7$ cups/day: $1305 \mathrm{~mL}$ & \multirow[b]{2}{*}{0.03} \\
\hline & Men & $\begin{array}{c}721 \\
0.55(0.51,0.59)\end{array}$ & $\begin{array}{c}1145 \\
0.53(0.50,0.56)\end{array}$ & $\begin{array}{c}1986 \\
0.51(0.49,0.53)\end{array}$ & $\begin{array}{c}469 \\
0.5(0.46,0.55)\end{array}$ & $\begin{array}{c}86 \\
0.44(0.35,0.55)\end{array}$ & \\
\hline & Women & $\begin{array}{c}892 \\
0.40(0.38,0.43)\end{array}$ & $\begin{array}{c}1578 \\
0.40(0.38,0.42)\end{array}$ & $\begin{array}{c}2944 \\
0.39(0.38,0.41)\end{array}$ & $\begin{array}{c}444 \\
0.41(0.37,0.45)\end{array}$ & $\begin{array}{c}60 \\
0.31(0.24,0.39)\end{array}$ & 0.50 \\
\hline \multirow{3}{*}{ ATTICA study [17] } & & None: $0 \mathrm{~mL}$ & $<200 \mathrm{~mL} /$ day: $100 \mathrm{~mL}$ & $\begin{array}{c}200-400 \text { mL/day: } 300 \\
\mathrm{~mL}\end{array}$ & >400 mL/day: 650 mL & - & \multirow[b]{2}{*}{0.11} \\
\hline & Men & $\begin{array}{c}133 \\
2.3(0.76,3.84)\end{array}$ & $\begin{array}{c}758 \\
2.2(0.76,3.64)\end{array}$ & $\begin{array}{c}521 \\
2.9(-0.56,6.36)\end{array}$ & $\begin{array}{c}27 \\
3.1(1.85,4.35)\end{array}$ & - & \\
\hline & Women & $\begin{array}{c}366 \\
2.1(0.66,3.54)\end{array}$ & $\begin{array}{c}922 \\
2.0(0.56,3.44)\end{array}$ & $\begin{array}{c}211 \\
2.7(-0.76,6.16)\end{array}$ & $\begin{array}{c}19 \\
2.9(1.36,4.44)\end{array}$ & - & 0.21 \\
\hline
\end{tabular}


Table 2. Cont.

\begin{tabular}{|c|c|c|c|c|c|c|c|}
\hline \multirow{3}{*}{ Study Name } & \multirow{3}{*}{ Gender } & \multicolumn{5}{|c|}{ Categories in the Original Study: Median or Mid-Point Volume $(\mathrm{mL})$} & \multirow{3}{*}{$P$-Trend * } \\
\hline & & None & Low & Medium & High & Very High & \\
\hline & & \multicolumn{5}{|c|}{$\begin{array}{l}\text { Number of Participants } \\
\text { Geometric Mean c-Reactive Protein (95\% Confidence Intervals) (mg/L) }\end{array}$} & \\
\hline \multirow{2}{*}{$\begin{array}{c}\text { Singapore } \\
\text { Prospective Study } \\
{[19]}\end{array}$} & & Never/rarely: $0 \mathrm{~mL}$ & $<1$ cup/day: $123 \mathrm{~mL}$ & $1-2$ cups/day: $323 \mathrm{~mL}$ & $\geq 3$ cups/day: $860 \mathrm{~mL}$ & - & \multirow[b]{2}{*}{0.37} \\
\hline & Both & $\begin{array}{c}1202 \\
1.31(1.16,1.49)\end{array}$ & $\begin{array}{c}475 \\
1.43(1.25,1.65)\end{array}$ & $\begin{array}{c}2118 \\
1.28(1.14,1.44)\end{array}$ & $\begin{array}{c}344 \\
1.23(1.05,1.44)\end{array}$ & - & \\
\hline \multirow{2}{*}{$\begin{array}{l}\text { Aichi Workers' } \\
\text { Cohort Study [20] }\end{array}$} & & $<1$ cup: $75 \mathrm{~mL}$ & 1 cup: $150 \mathrm{~mL}$ & $2-3$ cups: $375 \mathrm{~mL}$ & $\geq 4$ cups/day: $750 \mathrm{~mL}$ & - & \multirow[b]{2}{*}{0.51} \\
\hline & Both & $\begin{array}{c}949 \\
0.43(0.41,0.47)\end{array}$ & $\begin{array}{c}803 \\
0.4(0.37,0.43)\end{array}$ & $\begin{array}{c}1336 \\
0.37(0.35,0.40)\end{array}$ & $\begin{array}{c}229 \\
0.42(0.36,0.48)\end{array}$ & - & \\
\hline \multirow{2}{*}{$\begin{array}{l}\text { United Kingdom } \\
\text { study [21] }\end{array}$} & & - & $<1$ cup/day: $45 \mathrm{~mL}$ & 1-2 cups/day: $195 \mathrm{~mL}$ & $>2$ cups/day: $435 \mathrm{~mL}$ & - & \multirow[b]{2}{*}{0.94} \\
\hline & Men & - & $\begin{array}{c}16 \\
0.97(0.77,1.17)\end{array}$ & $\begin{array}{c}20 \\
0.83(0.64,1.02)\end{array}$ & $\begin{array}{c}41 \\
0.94(0.82,1.06)\end{array}$ & - & \\
\hline \multirow{2}{*}{$\begin{array}{l}\text { FinnDiane Study } \\
{[23]}\end{array}$} & & $<1$ cup/day: $75 \mathrm{~mL}$ & $\begin{array}{l}\geq 1 \text { cup/day }<3: 300 \\
m L\end{array}$ & $\begin{array}{l}\geq 3 \text { cups } / \text { day }<5: 600 \\
m L\end{array}$ & $\geq 5$ cups/day: $1013 \mathrm{~mL}$ & - & \multirow[b]{2}{*}{0.27} \\
\hline & Both & $\begin{array}{c}134 \\
1.93(1.56,2.30)\end{array}$ & $\begin{array}{c}230 \\
1.88(1.61,2.16)\end{array}$ & $\begin{array}{c}371 \\
1.62(1.41,1.82)\end{array}$ & $\begin{array}{c}305 \\
1.68(1.44,1.91)\end{array}$ & - & \\
\hline \multirow{2}{*}{ BELSTRESS [24] } & & None & 1-3 cups/day: $300 \mathrm{~mL}$ & >3cups/day: 750 mL & - & - & \multirow[b]{2}{*}{0.30} \\
\hline & Men & $\begin{array}{c}168 \\
0.89(0.75,1.04)\end{array}$ & $\begin{array}{c}415 \\
0.95(0.81,1.11)\end{array}$ & $\begin{array}{c}448 \\
0.97(0.82,1.14)\end{array}$ & - & - & \\
\hline
\end{tabular}

* $P$-trend was re-calculated based on the estimated mid-point volume (in $\mathrm{mL}$ ) of coffee consumption in each category and obtained by treating coffee volume as continuous in a model testing a linear association between coffee consumption and CRP level. 


\section{Discussion}

To our knowledge, this is the first dose-response meta-analysis of the association between coffee consumption and CRP level in cross-sectional analyses that considered the volume of coffee consumed instead of categorical data, as employed in a previous meta-analysis [11]. Hence, our analysis is more robust in assessing the effects of volume of coffee consumed, which reflects the amount of bioactive compounds in coffee better than considering categories alone [12-14]. This is important as cup volume varied across studies (150 to $237 \mathrm{~mL}$ ). When studies were combined through the dose-response meta-analysis, no statistically significant associations were observed between coffee consumption and CRP levels among all studies or after stratifying by gender or geographic location. To further elucidate this finding, we examined associations of CRP level with coffee consumption by modeling the volume of coffee and re-calculating the $p$-value for linear associations for each study. We found that the three studies with the largest sample sizes, NHS, EPIC study, and Kyushu University cohort, had statistically significant inverse or positive associations between coffee consumption and CRP levels, while others reported no statistically significant association. These inconsistent associations across studies may be explained by differences in study populations as discussed below.

The characteristics related to coffee preference and preparation methods common in each study population may have affected the associations between coffee consumption and CRP level due to variation in the amount of bioactive compounds $[12,13,47,48]$. Around the time when coffee consumption was assessed in the included countries, instant coffees were popular in all countries. Unfiltered coffee was more commonly consumed in European countries than in the United States, Japan, and Singapore, where filtered coffee was more common [49]. Among the 11 included studies, three investigated associations separately by coffee preparation methods or types-filtered and unfiltered coffees in the ATTICA study [17] and decaffeinated and caffeinated coffees in the NHS and HPFS [18]. However, all studies found similar associations for either method [17,18]. Moreover, previous coffee intervention trials reported no difference in CRP levels by different types or preparation methods of coffee $[34,50,51]$. Nevertheless, a previous animal study provided evidence of anti-inflammatory effects of caffeine, given that a three-week caffeine administration ( 7.5 to $15 \mathrm{mg} / \mathrm{kg}$ of body weight) resulted in decreased CRP level in rats [7]. Therefore, previous human studies [34,50,51] may not have had a sufficiently large variation in coffee consumption (due to small sample sizes or homogeneous coffee consumption within a single study population) to observe differences in CRP levels comparable to the animal study [7]. Our meta-analysis had a limited ability to further explore associations by coffee preparation methods or types given that only three of the included studies conducted separate analyses $[17,18]$. In addition to caffeine, chlorogenic acid was reported to decrease inflammation in vitro, and its content varies by roasting levels [52]. To provide greater insight, future human studies may need to assess type, preparation methods and roasting types, along with the volume of coffee consumed.

In addition, discrepant results between studies could be due to participants' characteristics common in groups defined by gender and geographic location. These characteristics might have contributed to their interactions with coffee consumption or have confounded the observed associations. Firstly, BMI may have affected the inconsistent results between European men and women and Japanese men. Given the pro-inflammatory nature of CRP, a positive association of CRP levels with BMI and body fat composition was reported previously [53]. The EPIC study, with a positive association, had a higher average BMI $\left(26.3 \mathrm{~kg} / \mathrm{m}^{2}\right)$ [44] than the Kyushu University cohort $\left(23.5 \mathrm{~kg} / \mathrm{m}^{2}\right)$ [25], which reported an inverse association. Similarly, within the same EPIC study population, opposing associations (a positive association for men and an inverse association for women) were reported, which suggests that BMI $\left(26.3 \mathrm{~kg} / \mathrm{m}^{2}\right.$ for men and $24.2 \mathrm{~kg} / \mathrm{m}^{2}$ for women on average) [44] might have played a role rather than other factors such as coffee type. Hence, it is possible that BMI or body fat mass may have contributed to conflicting associations of coffee consumption with CRP levels in the EPIC and Kyushu University cohort studies. To further elucidate potential involvement of body fat 
mass, future studies need to use other anthropometric measures that are more reflective of body fat mass than BMI and stratify results by these anthropometric factors.

Secondly, smoking might have contributed to discrepant results. CRP levels were higher among smokers than non-smokers [54], and smoking tends to be more common among men than women, especially in Japan $[25,55]$ and Singapore [56]. In addition, confounding effects of smoking in the association between coffee and CRP level are possible as previously reported for coronary artery disease or CVD mortality [3,57], which were closely linked to CRP levels. For the studies with statistically significant associations, the proportion of current smokers was 32\% for men in the Kyushu University cohort [25], 30\% for men and 17\% for women in the EPIC study [45], and 13\% in the NHS [18]. Hence, the relatively low proportion of current smokers might partially explain the inverse association for European and US women. However, other factors may play a role in conflicting associations between European and Japanese men, which warrants further investigations.

Alcohol intake may have also been an influential variable in gender differences with regard to the association between coffee consumption and CRP level. Among women, studies with a significant inverse association tended to have a higher alcohol consumption ( $6.0 \mathrm{~g} /$ day in NHS [18] and $4.2 \mathrm{~g} /$ day in EPIC women [22]) than studies with a non-significant association (1.7 g/day in DREW trial [26] and $27.1 \%$ as alcohol consumers in women in Kyushu University Cohort [25]). Among men, however, this trend was not clearly observed; EPIC men with a significant positive association reported consumption of $14.5 \mathrm{~g} /$ day [22]; Kyushu University Cohort men with a significant inverse association reported $72.7 \%$ of current alcohol consumers [25]; other studies with non-significant associations reported consumption in the range of $11.8 \mathrm{~g} /$ day in HPFS [18] and $27.3 \mathrm{~g} /$ day in BELSTRESS [24]. Among men in the Kyushu University Cohort, the inverse association between coffee and CRP was strongest among high current alcohol consumers [25], which might have driven the overall inverse association when all men in this study were combined. In the EPIC study, the association between coffee and CRP was not reported by alcohol consumption level. Biological effects may be influenced by consumption differences between genders, suggesting that the inverse association may be stronger for drinkers than non-drinkers. The positive association observed in EPIC men [22] may be partially explained by a previously reported U-shaped association between alcohol consumption and CRP [58], which warrants further investigation. The stronger inverse association between coffee and CRP in high alcohol consumers among Japanese men [25] is not consistent with the reported U-shaped association; however, relatively lower BMI in Japanese men than European men might have contributed to this difference. Additionally, the proportion of smokers was similar between Japanese and European men, which may not explain the opposing associations reported. Taken together, other potential factors (e.g., types of alcoholic beverages) that may explain the discrepant associations between European and Japanese men need to be explored in future studies.

Strengths of this current analysis include the large number of participants (a total of 61,047), with a majority of the included studies comprising over 500 participants. This analysis included a diverse population of apparently healthy men and women, overweight and postmenopausal women, and men and women with known diabetes or metabolic syndrome. Additionally, this analysis obtained results from over 15 different countries in Europe, North America, and Asia. This diversity in study populations allowed us to cover a wide range of coffee consumption levels (the estimated median volume of coffee consumed ranged from $150 \mathrm{~mL}$ in Aichi Workers' cohort [20] to $570 \mathrm{~mL}$ in the FinnDiane study [23]) that could not be achieved within a single study, and it allowed us to conduct a thorough examination of the association between coffee consumption and CRP levels. We also estimated the volume of coffee consumed in our analysis, instead of the pre-defined category or number of cups consumed, which better reflects the amount of hypothesized bioactive compounds in coffee. In addition, the comparability of CRP values across studies is a strength as all studies measured high-sensitivity CRP. The duration of blood sample storage varied by study; however, CRP values were reported to be highly stable over time (spanning several years) when blood samples were stored under the well-kept conditions [59] that all included studies followed. There may be slight variations 
in blood collection and handling procedures (such as temperature or time between blood collection and storage), which were also reported not to affect CRP values [60].

A limitation of this analysis is that the sample size ranged from 61 to 15,551, although a majority of the studies had over 500 participants. This may have affected the number of coffee consumption categories and statistical power within a single study. Hence, we conducted a dose-response meta-analysis including all eligible studies. Secondly, regarding CRP levels, two studies of women in the United States (i.e., DREW trial and NHS) had relatively higher levels than the rest of the studies. Previously, obesity and hormone therapy use were reported as determinants of high CRP level $[38,53,61]$. Both studies $[18,26]$ had the two highest proportions of hormone therapy users (46.5\% and $41.3 \%$ for the DREW trial and NHS, respectively), and the DREW trial included only overweight and obese women, while the NHS had a higher average BMI than other studies; these two characteristics may explain their high CRP levels. Thirdly, except for sex, we had limited ability to explore effects of potential confounding factors such as smoking and BMI. This is due to the fact that we did not have participant-level data and we relied on the reported associations adjusted for a set of confounding variables chosen by study investigators. Moreover, our study was limited in exploring the confounding effect of age because age ranges of study participants overlapped among the included studies and no study conducted analyses stratified by age group. Fourthly, all studies were based on cross-sectional analyses, which cannot infer temporal association, and future prospective analyses are warranted. Fifthly, our analysis only included one biomarker of chronic inflammation, CRP. Thus, other biomarkers such as interleukin-6 and tumor necrosis factor-alpha need to be explored in future studies. These biomarkers were linked to coffee extracts in previous in vitro [52] and human studies $[17,18]$. Furthermore, there is recent development in isoforms of CRP, such as pentameric and monomeric isoforms, linked to cardiovascular diseases and inflammatory conditions [62], which need to be considered in future studies. Sixthly, all the included studies used a food frequency questionnaire (FFQ) to assess coffee consumption. Although most were previously validated and reported correlation coefficients of coffee intake between FFQ and other dietary assessment instruments as high as 0.78 [31], the use of FFQs may have impacted the coffee consumption data due to inherent self-reporting errors [63]. Nonetheless, it is an efficient way to assess dietary intake in a large sample size. Future studies could use biomarkers of coffee consumption (e.g., urinary furoylglycine [64,65], $N$-methylpyridinium, and trigonelline [66]) to overcome limitations in self-reporting methods. Additionally, these biomarker studies would address the potential difference in the amount of bioactive compounds resulting from differences in coffee preparation, brew strength, roasting and beans, which cannot be construed solely by the volume of coffee consumed. Future studies measuring biomarkers of coffee consumption or collecting detailed information on coffee type and preparation method are warranted.

\section{Conclusions}

Our results from the dose-response meta-analysis suggest no statistically significant association between coffee consumption and CRP level among all studies combined or after stratification by gender and geographic location. The three individual studies with the largest sample sizes among the 11 included studies support an inverse or positive association between coffee consumption and CRP levels among European men and women, US women, and Japanese men. Given these conflicting associations, factors such as smoking and BMI may be attributable to these variations, and the potential interaction with gender needs to be explored further.

Supplementary Materials: The following are available online at http://www.mdpi.com/2072-6643/12/5/1349/s1, Table S1: Risk of Bias Assessment.

Author Contributions: Conceptualization, E.D.M. and Y.T.; methodology, Y.T. and C.H.; formal analysis C.H.; writing-Original draft preparation, E.D.M. and Y.T.; writing-Review and editing, C.H., N.D., and N.G.H.; supervision, Y.T.; project administration, Y.T. All authors have read and agreed to the published version of the manuscript.

Funding: This study received no funding. 
Acknowledgments: E.D.M. completed this work as part of her Honors' thesis in the College of Public Health and Human Sciences at Oregon State University.

Conflicts of Interest: None declared by all authors (E.D.M., C.H., N.D., N.G.H., and Y.T.).

$\begin{array}{ll}\text { Abbreviations } \\ \text { BMI } & \text { body mass index } \\ \text { CI } & \text { confidence intervals } \\ \text { CRP } & \text { c-reactive protein } \\ \text { CVD } & \text { cardiovascular disease } \\ \text { DREW } & \text { Dose-Response to Exercise in Women } \\ \text { EPIC } & \text { European Prospective Investigation into Cancer and Nutrition } \\ \text { FFQ } & \text { food frequency questionnaire } \\ \text { FinnDiane } & \text { Finnish Diabetic Nephropathy } \\ \text { HPFS } & \text { Health Professional Follow-Up Study } \\ \text { NHS } & \text { Nurses' Health Study } \\ \text { UK } & \text { United Kingdom } \\ \text { US } & \text { United States }\end{array}$

\section{References}

1. Frost-Meyer, N.J.; Logomarsino, J.V. Impact of coffee components on inflammatory markers: A review. J. Funct. Foods 2012, 4, 819-830. [CrossRef]

2. Nieber, K. The Impact of Coffee on Health. Planta Med. 2017. [CrossRef]

3. Grosso, G.; Micek, A.; Godos, J.; Sciacca, S.; Pajak, A.; Martinez-Gonzalez, M.A.; Giovannucci, E.L.; Galvano, F. Coffee consumption and risk of all-cause, cardiovascular, and cancer mortality in smokers and non-smokers: A dose-response meta-analysis. Eur. J. Epidemiol. 2016, 31, 1191-1205. [CrossRef]

4. Rodrigues, I.M.; Klein, L.C. Boiled or filtered coffee? Effects of coffee and caffeine on cholesterol, fibrinogen and C-reactive protein. Toxicol. Rev. 2006, 25, 55-69. [CrossRef]

5. Ansar, W.; Ghosh, S. C-reactive protein and the biology of disease. Immunol. Res. 2013, 56, 131-142. [CrossRef]

6. Santana-Galvez, J.; Cisneros-Zevallos, L.; Jacobo-Velazquez, D.A. Chlorogenic Acid: Recent Advances on Its Dual Role as a Food Additive and a Nutraceutical against Metabolic Syndrome. Molecules 2017, 22, 358. [CrossRef]

7. Owoyele, B.V.; Oyewole, A.L.; Biliaminu, S.A.; Alashi, Y. Effect of taurine and caffeine on plasma c-reactive protein and calcium in Wistar rats. Afr. J. Med. Med. Sci. 2015, 44, 229-236. [PubMed]

8. Kim, H.G.; Kim, J.Y.; Hwang, Y.P.; Lee, K.J.; Lee, K.Y.; Kim, D.H.; Kim, D.H.; Jeong, H.G. The coffee diterpene kahweol inhibits tumor necrosis factor-alpha-induced expression of cell adhesion molecules in human endothelial cells. Toxicol. Appl. Pharmacol. 2006, 217, 332-341. [CrossRef]

9. Bak, A.A.; Grobbee, D.E. The effect on serum cholesterol levels of coffee brewed by filtering or boiling. N. Engl. J. Med. 1989, 321, 1432-1437. [CrossRef]

10. Zock, P.L.; Katan, M.B.; Merkus, M.P.; van Dusseldorp, M.; Harryvan, J.L. Effect of a lipid-rich fraction from boiled coffee on serum cholesterol. Lancet 1990, 335, 1235-1237. [CrossRef]

11. Zhang, Y.; Zhang, D.Z. Is coffee consumption associated with a lower level of serum C-reactive protein? A meta-analysis of observational studies. Int. J. Food Sci. Nutr. 2018. [CrossRef]

12. Arisseto, A.P.; Vicente, E.; Ueno, M.S.; Tfouni, S.A.; Toledo, M.C. Furan levels in coffee as influenced by species, roast degree, and brewing procedures. J. Agric. Food Chem. 2011, 59, 3118-3124. [CrossRef]

13. Moon, J.K.; Shibamoto, T. Role of roasting conditions in the profile of volatile flavor chemicals formed from coffee beans. J. Agric. Food Chem. 2009, 57, 5823-5831. [CrossRef]

14. Yanagimoto, K.; Ochi, H.; Lee, K.G.; Shibamoto, T. Antioxidative activities of fractions obtained from brewed coffee. J. Agric. Food Chem. 2004, 52, 592-596. [CrossRef]

15. Moher, D.; Liberati, A.; Tetzlaff, J.; Altman, D.G.; Group, P. Preferred reporting items for systematic reviews and meta-analyses: The PRISMA statement. PLoS. Med. 2009, 6, e1000097. [CrossRef] 
16. Paiva, C.; Beserra, B.; Reis, C.; Dorea, J.G.; Da Costa, T.; Amato, A.A. Consumption of coffee or caffeine and serum concentration of inflammatory markers: A systematic review. Crit. Rev. Food Sci. Nutr. 2019, 59, 652-663. [CrossRef]

17. Zampelas, A.; Panagiotakos, D.B.; Pitsavos, C.; Chrysohoou, C.; Stefanadis, C. Associations between coffee consumption and inflammatory markers in healthy persons: The ATTICA study. Am. J. Clin. Nutr. 2004, 80, 862-867. [CrossRef]

18. Hang, D.; Kvaerner, A.S.; Ma, W.; Hu, Y.; Tabung, F.K.; Nan, H.; Hu, Z.; Shen, H.; Mucci, L.A.; Chan, A.T.; et al. Coffee consumption and plasma biomarkers of metabolic and inflammatory pathways in US health professionals. Am. J. Clin. Nutr. 2019, 109, 635-647. [CrossRef]

19. Rebello, S.A.; Chen, C.H.; Naidoo, N.; Xu, W.; Lee, J.; Chia, K.S.; Tai, E.S.; van Dam, R.M. Coffee and tea consumption in relation to inflammation and basal glucose metabolism in a multi-ethnic Asian population: A cross-sectional study. Nutr. J. 2011, 10, 61. [CrossRef]

20. Yamashita, K.; Yatsuya, H.; Muramatsu, T.; Toyoshima, H.; Murohara, T.; Tamakoshi, K. Association of coffee consumption with serum adiponectin, leptin, inflammation and metabolic markers in Japanese workers: A cross-sectional study. Nutr. Diabetes 2012, 2, e33. [CrossRef]

21. Hamer, M.; Williams, E.D.; Vuononvirta, R.; Gibson, E.L.; Steptoe, A. Association between coffee consumption and markers of inflammation and cardiovascular function during mental stress. J. Hypertens. 2006, 24, 2191-2197. [CrossRef]

22. Gunter, M.J.; Murphy, N.; Cross, A.J.; Dossus, L.; Dartois, L.; Fagherazzi, G.; Kaaks, R.; Kuhn, T.; Boeing, H.; Aleksandrova, K.; et al. Coffee Drinking and Mortality in 10 European Countries: A Multinational Cohort Study. Ann. Intern. Med. 2017, 167, 236-247. [CrossRef]

23. Stutz, B.; Ahola, A.J.; Harjutsalo, V.; Forsblom, C.; Groop, P.H.; FinnDiane Study, G. Association between habitual coffee consumption and metabolic syndrome in type 1 diabetes. Nutr. Metab. Cardiovasc. Dis. 2018, 28, 470-476. [CrossRef]

24. De Bacquer, D.; Clays, E.; Delanghe, J.; De Backer, G. Epidemiological evidence for an association between habitual tea consumption and markers of chronic inflammation. Atherosclerosis 2006, 189, 428-435. [CrossRef]

25. Maki, T.; Pham, N.M.; Yoshida, D.; Yin, G.; Ohnaka, K.; Takayanagi, R.; Kono, S. The relationship of coffee and green tea consumption with high-sensitivity C-reactive protein in Japanese men and women. Clin. Chem. Lab. Med. 2010, 48, 849-854. [CrossRef]

26. Arsenault, B.J.; Earnest, C.P.; Despres, J.P.; Blair, S.N.; Church, T.S. Obesity, coffee consumption and CRP levels in postmenopausal overweight/obese women: Importance of hormone replacement therapy use. Eur. J. Clin. Nutr. 2009, 63, 1419-1424. [CrossRef]

27. Herzog, R.; Alvarez-Pasquin, M.J.; Diaz, C.; Del Barrio, J.L.; Estrada, J.M.; Gil, A. Are healthcare workers' intentions to vaccinate related to their knowledge, beliefs and attitudes? A systematic review. BMC Public Health 2013, 13, 154. [CrossRef]

28. Higgins, J.P.; Thompson, S.G. Quantifying heterogeneity in a meta-analysis. Stat. Med. 2002, 21, 1539-1558. [CrossRef]

29. Pham, N.M.; Wang, Z.; Morita, M.; Ohnaka, K.; Adachi, M.; Kawate, H.; Takayanagi, R.; Kono, S. Combined effects of coffee consumption and serum gamma-glutamyltransferase on serum C-reactive protein in middle-aged and elderly Japanese men and women. Clin. Chem. Lab. Med. 2011, 49, 1661-1667. [CrossRef]

30. Jacobs, S.; Kroger, J.; Floegel, A.; Boeing, H.; Drogan, D.; Pischon, T.; Fritsche, A.; Prehn, C.; Adamski, J.; Isermann, B.; et al. Evaluation of various biomarkers as potential mediators of the association between coffee consumption and incident type 2 diabetes in the EPIC-Potsdam Study. Am. J. Clin. Nutr. 2014, 100, 891-900. [CrossRef]

31. Lopez-Garcia, E.; van Dam, R.M.; Qi, L.; Hu, F.B. Coffee consumption and markers of inflammation and endothelial dysfunction in healthy and diabetic women. Am. J. Clin. Nutr. 2006, 84, 888-893. [CrossRef]

32. Williams, C.J.; Fargnoli, J.L.; Hwang, J.J.; van Dam, R.M.; Blackburn, G.L.; Hu, F.B.; Mantzoros, C.S. Coffee consumption is associated with higher plasma adiponectin concentrations in women with or without type 2 diabetes: A prospective cohort study. Diabetes Care 2008, 31, 504-507. [CrossRef]

33. Martinez-Lopez, S.; Sarria, B.; Mateos, R.; Bravo-Clemente, L. Moderate consumption of a soluble green/roasted coffee rich in caffeoylquinic acids reduces cardiovascular risk markers: Results from a randomized, cross-over, controlled trial in healthy and hypercholesterolemic subjects. Eur J. Nutr. 2018. [CrossRef] 
34. Kempf, K.; Kolb, H.; Gartner, B.; Bytof, G.; Stiebitz, H.; Lantz, I.; Lang, R.; Hofmann, T.; Martin, S. Cardiometabolic effects of two coffee blends differing in content for major constituents in overweight adults: A randomized controlled trial. Eur. J. Nutr. 2015, 54, 845-854. [CrossRef]

35. Hu, G.; Jousilahti, P.; Tuomilehto, J.; Antikainen, R.; Sundvall, J.; Salomaa, V. Association of serum C-reactive protein level with sex-specific type 2 diabetes risk: A prospective finnish study. J. Clin. Endocrinol. Metab. 2009, 94, 2099-2105. [CrossRef]

36. Raaska, K.; Raitasuo, V.; Laitila, J.; Neuvonen, P.J. Effect of caffeine-containing versus decaffeinated coffee on serum clozapine concentrations in hospitalised patients. Basic Clin. Pharmacol. Toxicol. 2004, 94, 13-18. [CrossRef]

37. Kotani, K.; Sakane, N.; Yamada, T.; Taniguchi, N. Association between coffee consumption and the estimated glomerular filtration rate in the general Japanese population: Preliminary data regarding C-reactive protein concentrations. Clin. Chem. Lab. Med. 2010, 48, 1773-1776. [CrossRef]

38. de Maat, M.P.; Kluft, C. Determinants of C-reactive protein concentration in blood. Ital. Heart J. 2001, 2, 189-195. [CrossRef]

39. Coffee linked to increased levels of heart risk markers. Health News 2005, 11, 15.

40. Hamer, M.; Williams, E.; Vuonovirta, R.; Giacobazzi, P.; Gibson, E.L.; Steptoe, A. The effects of effort-reward imbalance on inflammatory and cardiovascular responses to mental stress. Psychosom Med. 2006, 68, 408-413. [CrossRef]

41. Ahola, A.J.; Lassenius, M.I.; Forsblom, C.; Harjutsalo, V.; Lehto, M.; Groop, P.H. Dietary patterns reflecting healthy food choices are associated with lower serum LPS activity. Sci. Rep. 2017, 7, 6511. [CrossRef]

42. Ahola, A.J.; Mikkila, V.; Makimattila, S.; Forsblom, C.; Freese, R.; Groop, P.H.; FinnDiane Study, G. Energy and nutrient intakes and adherence to dietary guidelines among Finnish adults with type 1 diabetes. Ann. Med. 2012, 44, 73-81. [CrossRef]

43. Moreau, M.; Valente, F.; Mak, R.; Pelfrene, E.; de Smet, P.; De Backer, G.; Kornitzer, M. Obesity, body fat distribution and incidence of sick leave in the Belgian workforce: The Belstress study. Int. J. Obes. Relat. Metab. Disord. 2004, 28, 574-582. [CrossRef]

44. McKenzie, F.; Biessy, C.; Ferrari, P.; Freisling, H.; Rinaldi, S.; Chajes, V.; Dahm, C.C.; Overvad, K.; Dossus, L.; Lagiou, P.; et al. Healthy Lifestyle and Risk of Cancer in the European Prospective Investigation Into Cancer and Nutrition Cohort Study. Medicine (Baltimore) 2016, 95, e2850. [CrossRef]

45. Ferrari, P.; Licaj, I.; Muller, D.C.; Kragh Andersen, P.; Johansson, M.; Boeing, H.; Weiderpass, E.; Dossus, L.; Dartois, L.; Fagherazzi, G.; et al. Lifetime alcohol use and overall and cause-specific mortality in the European Prospective Investigation into Cancer and nutrition (EPIC) study. BMJ Open 2014, 4, e005245. [CrossRef]

46. Verdaet, D.; Dendale, P.; De Bacquer, D.; Delanghe, J.; Block, P.; De Backer, G. Association between leisure time physical activity and markers of chronic inflammation related to coronary heart disease. Atherosclerosis 2004, 176, 303-310. [CrossRef]

47. Ratnayake, W.M.; Hollywood, R.; O'Grady, E.; Stavric, B. Lipid content and composition of coffee brews prepared by different methods. Food Chem. Toxicol. 1993, 31, 263-269. [CrossRef]

48. Smrke, S.; Opitz, S.E.; Vovk, I.; Yeretzian, C. How does roasting affect the antioxidants of a coffee brew? Exploring the antioxidant capacity of coffee via on-line antioxidant assays coupled with size exclusion chromatography. Food Funct. 2013, 4, 1082-1092. [CrossRef]

49. IARC Working Group. Monogr.: Eval. of Carcinogenic Risks to Humans: Coffee, Tea, Mate, Methylxanthines and Methylglyoxal; International Agency for Research on Cancer: Lyon, France, 1991.

50. Ohnaka, K.; Ikeda, M.; Maki, T.; Okada, T.; Shimazoe, T.; Adachi, M.; Nomura, M.; Takayanagi, R.; Kono, S. Effects of 16-week consumption of caffeinated and decaffeinated instant coffee on glucose metabolism in a randomized controlled trial. J. Nutr. Metab. 2012, 2012, 207426. [CrossRef]

51. Wedick, N.M.; Brennan, A.M.; Sun, Q.; Hu, F.B.; Mantzoros, C.S.; van Dam, R.M. Effects of caffeinated and decaffeinated coffee on biological risk factors for type 2 diabetes: A randomized controlled trial. Nutr. J. 2011, 10, 93. [CrossRef]

52. Jung, S.; Kim, M.H.; Park, J.H.; Jeong, Y.; Ko, K.S. Cellular Antioxidant and Anti-Inflammatory Effects of Coffee Extracts with Different Roasting Levels. J. Med. Food 2017, 20, 626-635. [CrossRef] [PubMed]

53. Kao, T.W.; Lu, I.S.; Liao, K.C.; Lai, H.Y.; Loh, C.H.; Kuo, H.K. Associations between body mass index and serum levels of C-reactive protein. SAMJ S. Afr. Med. J. 2009, 99, 326-330. [PubMed] 
54. Ahonen, T.M.; Kautiainen, H.J.; Keinanen-Kiukaanniemi, S.M.; Kumpusalo, E.A.; Vanhala, M.J. Gender Difference Among Smoking, Adiponectin, and High-Sensitivity C-Reactive Protein. Am. J. Prev. Med. 2008, 35, 598-601. [CrossRef] [PubMed]

55. Yamada, H.; Kawado, M.; Aoyama, N.; Hashimoto, S.; Suzuki, K.; Wakai, K.; Suzuki, S.; Watanabe, Y.; Tamakoshi, A.; Group, J.S. Coffee consumption and risk of colorectal cancer: The Japan Collaborative Cohort Study. J. Epidemiol. 2014, 24, 370-378. [CrossRef]

56. Ainslie-Waldman, C.E.; Koh, W.P.; Jin, A.; Yeoh, K.G.; Zhu, F.; Wang, R.; Yuan, J.M.; Butler, L.M. Coffee intake and gastric cancer risk: The Singapore Chinese health study. Cancer Epidemiol. Biomark. Prev. 2014, 23, 638-647. [CrossRef] [PubMed]

57. Klatsky, A.L.; Koplik, S.; Kipp, H.; Friedman, G.D. The confounded relation of coffee drinking to coronary artery disease. Am. J. Cardiol. 2008, 101, 825-827. [CrossRef]

58. Bell, S.; Mehta, G.; Moore, K.; Britton, A. Ten-year alcohol consumption typologies and trajectories of C-reactive protein, interleukin- 6 and interleukin-1 receptor antagonist over the following 12 years: A prospective cohort study. J. Intern. Med. 2017, 281, 75-85. [CrossRef]

59. Doumatey, A.P.; Zhou, J.; Adeyemo, A.; Rotimi, C. High sensitivity C-reactive protein (Hs-CRP) remains highly stable in long-term archived human serum. Clin. Biochem. 2014, 47, 315-318. [CrossRef]

60. Sugden, K.; Danese, A.; Shalev, I.; Williams, B.S.; Caspi, A. Blood Substrate Collection and Handling Procedures under Pseudo-Field Conditions: Evaluation of Suitability for Inflammatory Biomarker Measurement. Biodemography Soc. Biol. 2015, 61, 273-284. [CrossRef]

61. Sas, A.A.; Vaez, A.; Jamshidi, Y.; Nolte, I.M.; Kamali, Z.; T, D.S.; Riese, H.; Snieder, H. Genetic and environmental influences on stability and change in baseline levels of $\mathrm{C}$-reactive protein: A longitudinal twin study. Atherosclerosis 2017, 265, 172-178. [CrossRef]

62. Boncler, M.; Wu, Y.; Watala, C. The Multiple Faces of C-Reactive Protein-Physiological and Pathophysiological Implications in Cardiovascular Disease. Molecules 2019, 24, 2062. [CrossRef] [PubMed]

63. Subar, A.F.; Freedman, L.S.; Tooze, J.A.; Kirkpatrick, S.I.; Boushey, C.; Neuhouser, M.L.; Thompson, F.E.; Potischman, N.; Guenther, P.M.; Tarasuk, V.; et al. Addressing Current Criticism Regarding the Value of Self-Report Dietary Data. J. Nutr. 2015, 145, 2639-2645. [CrossRef]

64. Heinzmann, S.S.; Holmes, E.; Kochhar, S.; Nicholson, J.K.; Schmitt-Kopplin, P. 2-Furoylglycine as a Candidate Biomarker of Coffee Consumption. J. Agric. Food Chem. 2015, 63, 8615-8621. [CrossRef] [PubMed]

65. Stalmach, A.; Mullen, W.; Barron, D.; Uchida, K.; Yokota, T.; Cavin, C.; Steiling, H.; Williamson, G.; Crozier, A. Metabolite profiling of hydroxycinnamate derivatives in plasma and urine after the ingestion of coffee by humans: Identification of biomarkers of coffee consumption. Drug Metab. Dispos. 2009, 37, 1749-1758. [CrossRef] [PubMed]

66. Lang, R.; Wahl, A.; Stark, T.; Hofmann, T. Urinary N-methylpyridinium and trigonelline as candidate dietary biomarkers of coffee consumption. Mol. Nutr. Food Res. 2011, 55, 1613-1623. [CrossRef] 